\title{
Prevalência de acuidade visual reduzida nos alunos da primeir a série do en sino fundamental das redes pública estadual e privada de Londrina-PR, no ano de 2000
}

\author{
Reduced visual acuity prevalence in first grade school children of \\ elem entary state public and private schools of Londrina - PR,Brazil, in 2000
}

Gerson J orge A parecido Lopes ${ }^{1}$

Antônio Marcelo Barbante Casella²

Cristian e Assam i Chuí ${ }^{3}$

\section{RESUMO}

Objetivos: Verificar a prevalência de acuidade visual reduzida em estudantes da $1^{\underline{a}}$ série do ensino fundamental das redes pública estadual e privada de Londrina - PR, zona urbana; comparar essas prevalências; verificar e comparar a prevalência de escolares utilizando correção óptica por ocasião do teste de acuidade visual e descrever as causas da acuidade visual reduzida nessa população. Métodos: Teste de acuidade visual foi realizado em 1688 alunos das escolas públicas estaduais e 611 alunos das escolas privadas. As crianças com acuidade visual menor ou igual a 0,7 foram encaminhadas para exame oftalmológico. Resultados: A prevalência de acuidade visual reduzida foi de $17,1 \%$ na rede pública estadual e $19,8 \%$ na rede privada, cuja diferença não tem significância estatística. A prevalência de escolares usando óculos foi de $2,4 \%$ na rede estadual e de $3,6 \%$ na rede privada; sem diferença estatisticamente significativa entre estes resultados. As causas de acuidade visual reduzida foram erro refracional, ambliopia, estrabismo e outras causas. Conclusão: A prevalência de acuidade visual reduzida entre escolares das redes pública estadual e privada foi estatisticamente igual, bem como a prevalência de escolares utilizando correção óptica. As causas de acuidade visual reduzida foram erro refracional, ambliopia, estrabismo, afacia por catarata congênita, catarata congênita zonular, persistência de vítreo primário hiperplásico, palidez do nervo óptico e alteração macular.

Descritores: Acuidade visual; Estudos de prevalência; Saúde escolar; Educação em saúde

\section{INT R ODUÇÃO}

curso de Pós-graduação em Medicina da Universidade título de Mestre, pelo autor principal.

${ }^{1}$ Prof. Assistente - Mestre do Departamento de Clínica Cirúrgica da Universidade Estadual de Londrina, Disciplina de Oftalmologia.

2 Prof. Adjunto-Doutor do Departamento de Clínica Cirúrgica da Universidade Estadual de Londrina, Disciplina de Oftalmologia.

${ }^{3}$ Ex-residente de Oftalmologia do Hospital Universitário Regional Norte do Paraná da Universidade Estadual de Londrina.

Endereço para correspondência: Rua Guarapuava, 119 - Londrina (PR) CEP 86060-550.

E-mail: gerlopes@ sercomtel.com.br

Recebido para publicação em 05.09.2001

Aceito para publicação em 04.03.2002

Nota Editorial: Pela análise deste trabalho e por sua anuência sobre a divulgação desta nota, agradecemos ao Dr. Marinho Jorge Scarpi. 
Estima-se que a grande maioria das crianças brasileiras em idade escolar nunca passou por exame oftalmológico e dados do Conselho Brasileiro de Oftalmologia ${ }^{(7)}(\mathrm{CBO})$ mostram que $20 \%$ delas apresentam alguma perturbação ocular.

As causas mais comuns de A.V. reduzida em escolares são os erros de refração (a hipermetropia, o astigmatismo e a miopia), estrabismo e ambliopia. A detecção precoce de vícios de refração possibilita a sua correção ou minimização, visando o melhor rendimento global da criança em idade escolar ${ }^{(8)}$.

Nos programas de triagem visual é importante estipular o critério de encaminhamento dos indivíduos como, por exemplo, o limite de visão a ser considerado. Esta preocupação resulta do fato de que este não pode ser tão alto para que não haja um número excessivo de crianças encaminhadas, gerando exames desnecessários, bem como o contrário também é indesejável, pois pode deixar de lado crianças que tenham problemas oculares.

Um estudo para avaliar os critérios de triagem visual de escolares, concluiu que o limite de A.V. $\leq 0,7$ é conveniente em se tratando de Oftalmologia Sanitária; os autores enfatizam, também, que uma criança com visão de 0,8 , sem diferença de acuidade visual entre os olhos maior que duas linhas na tabela de optotipos, mesmo que necessitasse de correção óptica, não seria prejudicada pela falta de encaminhamento ${ }^{(2)}$.

Os objetivos deste estudo são verificar a prevalência de acuidade visual reduzida (A.V. $\leq 0,7)$ em escolares da primeira série do ensino fundamental de escolas das redes pública estadual e privada da cidade de Londrina - PR, zona urbana, no ano de 2000; comparar estas prevalências; verificar e comparar a prevalência de escolares utilizando correção óptica por ocasião do teste de A. V. e descrever as possíveis causas da baixa visual nesta população.

\section{MÉTODOS}

É um estudo transversal para detectar a prevalência de A.V. reduzida nos escolares da primeira série do ensino fundamental das redes pública estadual e privada da cidade de Londrina - PR, zona urbana, no ano de 2000.

Para chegar à prevalência de A.V. reduzida nos escolares, a pesquisa foi desenvolvida em três fases: triagem dos estudantes de $1^{\text {a }}$ série na própria escola realizada por professores treinados pelo NRE de Londrina (que desenvolve este trabalho há quatro anos); encaminhamento para exame oftalmológico das crianças que apresentaram acuidade visual reduzida; análise de dados.

Os métodos para a triagem de acuidade visual devem ser breves, simples, econômicos, eficazes e realizados por pessoas não especialistas, com o objetivo de indicar os casos que necessitem de exame oftalmológico especializado ${ }^{(1)}$.

Em contrapartida, outros autores reconhecem que nenhum dos métodos de triagem usado é perfeito e sempre vão aparecer falsos-positivos e falsos-negativos. Ainda segundo estes mesmos autores, a investigação de problemas oculares em crianças por médico oftalmologista é muito dispendiosa e inexeqüível do ponto de vista de Saúde Pública ${ }^{(2)}$. Portanto, o teste de acuidade visual deve ser realizado por profissional paramédico ou mesmo outra pessoa devidamente treinada e uma pessoa que poderia ser considerada ideal é justamente a figura do professor, conforme já observado anteriormente. Esta idéia é compartilhada por diversos autores ${ }^{(1-2,4,9-13)}$.

A validade do teste de visão realizada pelo professor ou outra pessoa treinada já foi motivo de estudo de diversos autores, que chegaram à conclusão de que é válido ${ }^{(1-2,9-10)}$.

$\mathrm{Na}$ escola, professores treinados mediram a A.V. através da tabela de optotipos "E" de Snellen a uma distância de seis metros, examinando cada olho separadamente, devendo a criança indicar as posições da letra "E". As crianças que usavam óculos foram examinadas sem e com óculos.

A amostra dimensionada para o estudo foi de 590 para um total de 1850 alunos matriculados nas escolas estaduais e de 454 estudantes entre os 952 matriculados na rede privada de ensino, da zona urbana de Londrina. Considerou-se a prevalência de $17 \%$, baseado em estudos já realizados em populações semelhantes, um erro na estimativa de $2,5 \%$ e nível de confiança de $95 \%$. Devido à estratégia de avaliação, foi possível avaliar 1778 alunos da rede estadual e 631 alunos da rede privada. Para efeito de cálculos do estudo, foram incluídos 1688 alunos da rede pública estadual e 611 estudantes da rede privada, pois foi excluída uma escola da rede pública estadual e uma escola da rede privada, por não terem seguido a metodologia padronizada para o teste de acuidade visual.

Quando o exame de triagem mostrava uma A.V. $\leq 0,7$, os pais ou responsáveis da criança receberam um comunicado esclarecendo a necessidade de submeter a criança a um exame oftalmológico completo.

Para a realização do exame oftalmológico foi colocado o consultório particular do primeiro autor deste trabalho à disposição daqueles que se interessassem, sem custos para os pais ou responsáveis. Para o exame dos escolares, o autor deste estudo contou com o auxílio de uma médica oftalmologista assistente. No caso do exame ter sido realizado por outro oftalmologista escolhido pelos pais ou responsáveis, foi solicitado ao médico que examinou a criança o preenchimento de um breve formulário, elaborado pelos autores desta pesquisa, contendo a A.V. sem e com correção, refração encontrada, fundo de olho e outras alterações oftalmológicas.

Efetivamente foram examinados $218(75,4 \%)$ alunos encaminhados das escolas estaduais e $84(69,4 \%)$ alunos das escolas particulares, sendo que $94,5 \%$ dos alunos da rede pública estadual e $77,3 \%$ da rede privada foram examinados no consultório do primeiro autor deste trabalho.

O exame oftalmológico consistiu em uma consulta oftalmológica padrão, que é realizada rotineiramente pelos médicos oftalmologistas. Este exame incluiu: exame externo, motilidade ocular, reflexo pupilar, acuidade visual sem e com correção, refração sob cicloplegia, fundoscopia direta e biomicroscopia (quando necessário). 
Nas crianças examinadas no consultório do primeiro autor deste estudo, a acuidade visual foi medida utilizando-se projetor de optotipos a uma distância de 6 metros. A cicloplegia foi obtida com colírio de tropicamida a $1 \%$, uma gota em cada olho de 10 em 10 minutos por duas vezes e o exame foi realizado 20 minutos após a última instilação. Caso a criança não fosse examinada em trinta minutos após a instilação do colírio, pingava-se uma nova gota, para manutenção do efeito cicloplégico ${ }^{(14)}$.

Os parâmetros de diagnósticos das alterações visuais e oculares adotados neste estudo foram baseados em diversos trabalhos publicados ${ }^{(8,15-20)}$, bem como nas instruções da Campanha Nacional de Reabilitação Visual desenvolvida pelo $\mathrm{CBO}^{(7)}$ :

- A.V.reduzida: A.V. $\leq 0,7$ em um dos olhos;

- Erros refracionais: crianças que necessitaram de correção óptica:

hipermetropia: $\geq+1,00 \mathrm{DE}$

miopia: $\geq-0,50 \mathrm{DE}$

astigmatismo: > 0,50 DC

anisometropia: diferença entre os dois olhos de $\geq 2,00$ dioptrias;

- Ambliopia: A.V. $\leq$ 0,7 corrigida em um dos olhos, sem lesão orgânica;

- Alteração ocular: presença de alteração ocular ou de anexo do olho, com ou sem comprometimento da visão, excluindo os erros refracionais e o estrabismo.

A prevalência de A.V. reduzida nas escolas estaduais e particulares foi estimada através do intervalo de confiança de $95 \%$.

A comparação das prevalências da rede pública estadual e privada foi realizada através do teste de Qui-quadrado com correção de Yates, em nível de significância de 5\% ( $\alpha=0,05)$, nas escolas estaduais e particulares.

O trabalho foi aprovado pelo Comitê de Ética em Pesquisa da Universidade Estadual de Londrina (UEL).

\section{RESULTADOS}

A tabela 1 mostra o número de escolas participantes e o número de alunos testados e incluídos no estudo.

Na rede pública estadual, dos 1688 alunos incluídos no estudo, 289 alunos apresentaram A.V. reduzida (A.V. $\leq 0,7)$ e foram encaminhados para exame oftalmológico. Na rede privada, dos

\begin{tabular}{|c|c|c|}
\hline Escolas/Alunos & Rede Estadual & Rede Privada \\
\hline Escolas & 35 & 33 \\
\hline Alunos matriculados & 1850 & 952 \\
\hline Escolas que realizaram o teste & 35 & 19 \\
\hline Alunos testados & 1778 & 631 \\
\hline Alunos incluídos no estudo & 1688 & 611 \\
\hline$\%$ de alunos incluídos no estudo & $91,2 \%$ & $64,1 \%$ \\
\hline
\end{tabular}

611 alunos incluídos no estudo, 121 estudantes apresentaram A.V.reduzida (A.V. $\leq 0,7$ ) e receberam encaminhamento.

A prevalência de A.V. reduzida foi de $17,1 \%$ na rede estadual e de $19,8 \%$ na rede particular. Não houve diferença estatisticamente significativa na comparação desta prevalência $(\mathrm{p}=0,154)$.

O número de crianças utilizando correção óptica por ocasião do teste de acuidade visual realizado na escola foi de 40 $(2,4 \%)$ alunos na rede estadual e de $22(3,6 \%)$ alunos na rede privada, sem diferença estatisticamente significativa $(\mathrm{p}=$ 0,143).

As causas de A.V. reduzida (A.V. $\leq 0,7)$ encontradas no exame oftalmológico foram: erro refracional, ambliopia, estrabismo e outras causas (como afacia por catarata congênita, catarata congênita zonular, vítreo primário hiperplásico, palidez do nervo óptico, coloboma do nervo óptico e alteração macular).

A prevalência de erro refracional foi de $37,6 \%$ na rede estadual e de $44,0 \%$ na rede privada.

Quanto à ambliopia, 8 alunos $(3,6 \%)$ das escolas estaduais a apresentaram e 5 alunos $(5,9 \%)$ das escolas particulares.

Estrabismo estava presente em 1 aluno $(0,46 \%)$ das escolas estaduais e em 2 alunos $(2,4 \%)$ das escolas particulares.

As outras causas de A.V. reduzida atingiram 5 crianças $(2,3 \%)$ da rede pública estadual e nenhuma criança da rede privada (Tabela 2 ).

O número de alunos que se submeteram ao exame oftalmológico pela primeira vez foi de 180 na rede estadual e de 47 na rede particular. Portanto, $82,5 \%$ dos alunos examinados das escolas estaduais e 55,9\% dos estudantes examinados das escolas privadas. Dois alunos na rede estadual e 6 alunos na rede privada não responderam se era ou não a primeira vez, e foram classificados como "não sei".

Dos alunos que eram submetidos ao exame pela primeira vez, $72(40 \%)$ da rede estadual e $17(36,1 \%)$ da rede particular apresentaram alteração visual.

\section{DISCUSSÃ O}

A relevância deste estudo deve-se aos vários aspectos contemplados na coleta e análise de dados: o levantamento de um índice, da cidade de Londrina, que até então era ignorado

\begin{tabular}{|c|c|c|c|c|}
\hline & $\begin{array}{l}\text { Rede Est } \\
(\mathrm{N}=21\end{array}$ & dual & $\begin{array}{r}\text { Rede Priv } \\
(\mathrm{N}=84\end{array}$ & ada \\
\hline & № de alunos & $\%$ & № de Alunos & $\%$ \\
\hline Erro refração & 82 & 37,6 & 37 & 44,0 \\
\hline Ambliopia & 8 & 3,6 & 5 & 5,9 \\
\hline Estrabismo & 1 & 0,4 & 2 & 2,4 \\
\hline Outras causas & 5 & 2,3 & - & - \\
\hline
\end{tabular}


pela comunidade científica; a inclusão de uma população - as escolas particulares - geralmente excluída em estudos do gênero; a conscientização dos grupos pedagógicos participantes da importância do instrumento de avaliação oftalmológica como ferramenta auxiliar no processo de aprendizagem; e por fim, a deteç̧ão de estudantes com deficiência visual, propiciando uma conscientização de problemas que, uma vez resolvidos, podem contribuir com melhoria de qualidade de vida e de performance escolar.

Em um estudo que envolveu 832 escolares de escola pública de nível primário em Cali - Colômbia, foi encontrado uma prevalência de $60,5 \%$ de transtornos visuais entre alunos repetentes, enquanto entre os não repetentes a taxa foi de apenas $12,1 \%$, uma associação estatisticamente significativa entre a prevalência de transtornos visuais e baixo rendimento escolar ${ }^{(21)}$.

Os vícios de refração interferem no bom rendimento escolar, pois em um estudo realizado com 270 crianças com suspeita de problema visual encaminhadas pelos professores para exame oftalmológico, $80,5 \%$ delas poderiam ter suas deficiências escolares explicadas por baixa visão e/ou vício de refração ${ }^{(22)}$.

A prevalência de A.V. reduzida de $17,1 \%$ na rede estadual e de $19,8 \%$ na rede privada, sem diferença estatisticamente significativa entre os dois grupos, comprovou uma das hipóteses levantadas: de que a prevalência na rede privada era a mesma da rede pública.

Estes resultados são semelhantes aos encontrados em outros trabalhos publicados na literatura no Brasil e no exterior, não obstante os critérios adotados e as amostras populacionais serem diferentes do presente estudo ${ }^{(4,12,16,19,21,23-25)}$.

Estudo com alunos da $1^{\mathrm{a}}$ a $8^{\mathrm{a}}$ séries do $1^{0}$ grau de duas escolas estaduais da cidade de São Paulo, demonstrou uma prevalência de A.V. reduzida de 15,98\% (A.V. < 0,8, ou seja, A.V. $\leq 0,7)$. Considerando apenas a $1^{\mathrm{a}}$ série este índice é menor do que o presente estudo: $11,46 \%{ }^{(4)}$.

Em um estudo com alunos da $1^{\mathrm{a}}$ a $4^{\mathrm{a}}$ séries do $1^{\circ} \mathrm{grau}$ em escolas de Porto Alegre - RS, foi encontrado 19\% de escolares com A.V. $\leq 0,66^{(19)}$.

A prevalência de A.V. reduzida (A.V. $\leq 0,7)$ e alterações oculares encontrada por em um estudo com pré-escolares e escolares da $1^{\mathrm{a}}$ a $8^{\mathrm{a}}$ séries de escolas municipais, estaduais e particulares da cidade de Ibiporã-PR, foi de $14,59 \%{ }^{(16)}$.

Entre pré-escolares e escolares de escolas estaduais e particulares, a prevalência de A.V. reduzida foi de $17,76 \%$ nas escolas estaduais e de $16,45 \%$ nas escolas particulares, sendo que não houve diferença estatisticamente significativa entre estas duas redes de ensino ${ }^{(23)}$.

Numa escola de alto padrão sócio-econômico de Curitiba PR, foi encontrado um índice menor do que o presente estudo, em comparação à rede privada: $13,9 \%$ de A.V. $\leq 0,7$ entre 129 crianças do $1^{0} \operatorname{grau}^{(12)}$.

Em um estudo desenvolvido no Chile, a prevalência foi de $15,8 \%$ de A.V. $\leq 0,5^{(24)}$. Na Colômbia, a prevalência foi de $19,6 \%$ de alunos com dificuldade visual (os autores não especificam o limite de a.v. ${ }^{(21)}$. Na Argentina foi encontrado uma prevalência de $18,69 \%$ de A.V. reduzida (A.V. $\leq 0,7)$ entre escolares do primeiro grau; entre os estudantes da primeira série a prevalência de A.V. reduzida foi de $18,09 \%{ }^{(25)}$.

Não se encontrou diferença estatisticamente significativa entre o número de alunos que já usavam óculos na ocasião do teste de A.V. realizado nas duas redes de ensino. Este resultado surpreendeu, pois se esperava um índice maior de crianças utilizando óculos na rede privada, já que se trata, teoricamente, de nível socioeconômico mais alto e, portanto, com mais facilidade de acesso aos serviços de saúde.

Esses índices são baixos se comparados com outros trabalhos publicados.

Em um estudo com 564 escolares da $1^{\underline{a}}$ a $4^{\underline{a}}$ séries do $1^{\underline{0}}$ grau, na cidade de Paulínia -SP, foi encontrado 9,2\% de crianças utilizando óculos ${ }^{20}$.

A prevalência de crianças usando óculos na ocasião do teste de A.V. em alunos da $1^{\text {a a }} 8^{\mathrm{a}}$ séries de duas escolas estaduais do município de São Paulo foi de 6,2\%. Já a prevalência encontrada na $1 \underline{\underline{a}}$ série foi de $1,04 \%$, resultado menor do que no presente estudo ${ }^{(4)}$.

Em um dos poucos estudos que envolveu a rede estadual e a rede privada, a prevalência de alunos usando óculos na rede estadual foi de $4,3 \%$ e de $9,3 \%$ na rede privada ${ }^{(23)}$.

No presente estudo, dos 289 alunos da rede estadual e dos 121 alunos da rede privada encaminhados para exame oftalmológico, foram examinados 218 e 84 respectivamente. Isso corresponde a um índice de $75,4 \%$ na rede estadual e $69,4 \%$ na rede privada. Portanto, a perda foi de $24,6 \%$ na rede pública estadual e de $30,6 \%$ na rede privada. Isto pode ser atribuído a uma das seguintes possibilidades: (i) os pais ou responsáveis não tomaram conhecimento da necessidade de submeter a criança ao exame oftalmológico; (ii) os pais ou responsáveis não levaram a criança ao médico; ou (iii) a criança foi examinada, mas o resultado não foi entregue à escola.

Em um estudo com pré-escolares da cidade de Campinas SP, o índice de não comparecimento foi de $56,4 \%$ dos casos encaminhados para exame oftalmológico ${ }^{(26)}$.

Em um outro estudo com pré-escolares, o índice foi de 20,6\% de não comparecimento das crianças triadas, mesmo depois de repetidos apelos feitos aos pais, segundo os autores ${ }^{(18)}$.

No Chile, em um trabalho desenvolvido com crianças de 5 a 15 anos, a taxa de perdas foi de $24,2 \%$, sendo que a taxa foi maior na classe socioeconômica mais alta ${ }^{(24)}$.

Dentre as crianças examinadas a A.V. reduzida foi confirmada em 88 alunos da rede estadual e em 38 alunos da rede privada. $\mathrm{Ou}$ seja, a taxa de verdadeiro-positivo foi de $40,3 \%$ e $45,2 \%$, respectivamente. Conseqüentemente, o falso-positivo foi de $59,7 \%$ na rede estadual e de $54,8 \%$ na rede privada, o que implicou num grande número de encaminhamentos desnecessários.

Isso pode ter ocorrido pela tendência dos(as) professores(as) de encaminhar os casos duvidosos ${ }^{(2,27)}$. Outro fator seria a curva de aprendizagem do teste de A.V. A criança chegava ao consultório oftalmológico conhecendo o teste de A.V. e, portanto, com uma possibilidade maior de fornecer a informação correta. 
A proporção de falso-negativo não foi quantificada, pois não se realizou exame em amostra de alunos que apresentaram A.V. normal no teste realizado pelo professor(a).

Os índices de falsos-positivos encontrados no presente estudo foram um pouco superiores aos apresentados por um estudo realizado em Ibiporã - PR e em Limeira - SP, onde os índices foram de $54,5 \%$ e de $49,3 \%$, respectivamente ${ }^{(16,27)}$.

Neste presente estudo, o resultado das causas de A.V. reduzida está de acordo com dados da literatura. A causa mais comum de A.V. reduzida foi o erro de refração, seguido da ambliopia e estrabismo.

O erro de refração foi encontrado em $37,6 \%$ dos alunos examinados da rede estadual e 44,0\% dos alunos examinados da rede privada. A ambliopia veio a seguir e foi observada em 8 alunos $(3,6 \%)$ das escolas estaduais e em 5 alunos $(5,9 \%)$ da rede privada. $\mathrm{O}$ estrabismo foi diagnosticado em 1 aluno $(0,4 \%)$ da rede estadual e em 2 alunos $(2,4 \%)$ da rede privada.

Estes índices, por si só, justificam a continuidade de programas de detecção de A.V. reduzida em escolares na cidade de Londrina, tanto na rede pública de ensino quanto na rede privada.

$O$ índice de erros refracionais entre 832 alunos de uma escola pública de nível primário na cidade de Cali - Colômbia, foi de $16,8 \%$ (21).

Em um estudo com crianças de 5 a 15 anos no Chile, o índice foi de $62,1 \%$ de erros refracionais como causa de A.V. reduzida $(\mathrm{A} . \mathrm{V} . \leq 0,5)^{(24)}$.

Entre alunos de 1a a 4a séries da cidade de São Paulo - SP, a prevalência de ametropias foi de $85,3 \%$. Os autores destacam que $78,2 \%$ dos casos eram de amétropes fisiológicos (pequenas ametropias que não necessitaram de correção $)^{(8)}$.

Em um trabalho com estudantes de 1aa a $8^{\underline{a}}$ séries de Porto Alegre - RS, foi encontrado 32,2\% de crianças que precisaram de óculos, ou seja, apresentaram erro refracional ${ }^{(19)}$.

$\mathrm{O}$ índice de ametropias entre pré-escolares e escolares da cidade de Ibiporã - PR foi de 31,2\%(16).

No presente estudo, a ambliopia foi diagnosticada em 8 alunos $(3,6 \%)$ da rede estadual e 5 alunos $(5,9 \%)$ da rede privada. Segundo o Conselho Brasileiro de Oftalmologia ${ }^{(7)}$, a prevalência de ambliopia entre escolares é em torno de 4\%. Mas esta prevalência varia entre os trabalhos publicados, depende da população estudada e dos critérios adotados ${ }^{(17-18,26,28)}$, portanto, a comparação entre os trabalhos fica prejudicada.

Outras alterações oculares com comprometimento visual, foram encontradas em 5 crianças $(2,3 \%$ do total de examinados) da rede estadual e em nenhuma criança da rede privada. A comparação deste dado com outros trabalhos fica prejudicada pela população e classificação diferentes utilizadas nos diversos estudos.

Neste estudo, 180 crianças $(82,5 \%)$ da rede estadual e 47 crianças $(55,9 \%)$ da rede privada estavam sendo submetidas ao exame oftalmológico pela primeira vez. Esses resultados são altos e mostram, mais uma vez, a necessidade de os pais ou responsáveis saberem que seu filho apresenta algum problema ocular para levá-los ao serviço especializado.
Dentre essas 180 crianças da rede estadual, 72 (40\%) apresentaram alteração visual. Na rede privada, 17 crianças $(36,1 \%)$, dentre as 47 , apresentaram o problema. Esses índices são significativos e mostram que os pais ou responsáveis não sabiam do problema visual de seu(sua) filho(a) e foi o teste de A.V. realizado na escola que detectou a A.V. reduzida. Isto corrobora a necessidade de se continuar a realização periódica do teste de A.V. em escolares, envolvendo todas as redes de ensino.

Além disso, há necessidade de conscientização dos pais ou responsáveis da importância de se submeter à criança a exame oftalmológico periodicamente, antes mesmo da idade escolar, independente da participação da escola. Outra medida que pode ser adotada é o esclarecimento dos pediatras sobre a necessidade de orientar os pais a levarem a criança ao médico oftalmologista para exame de rotina na idade pré-escolar.

Os resultados obtidos no presente estudo mostram que o programa de teste de A.V. em escolares é viável, efetivo e deve ter o seu espaço nos programas de saúde escolar, envolvendo as redes pública e privada de ensino.

\section{CONCLUSÃO}

- A prevalência de A.V. reduzida entre alunos da $1^{\mathrm{a}}$ série do ensino fundamental das redes pública estadual e privada de cidade de Londrina foi de $17,1 \%$ e $19,8 \%$, respectivamente e não tiveram diferença estatisticamente significativa.

- A prevalência de escolares utilizando óculos por ocasião do teste de A.V. foi de 2,4\% na rede estadual e de 3,6\% na rede privada, sem diferença estatisticamente significativa.

- As causas da A.V. reduzida nesta população foram: erro de refração, ambliopia, estrabismo, afacia por catarata congênita, catarata congênita zonular, persistência de vítreo primário hiperplásico, palidez do nervo óptico, alteração macular.

\section{A BST RACT}

Purpose: To analyze the prevalence of reduced visual acuity in students of elementary state public and private schools of Londrina's urban area; to compare the prevalence of reduced visual acuity in children of state public and private schools; to analyze and compare the prevalence of children wearing eyeglasses, and to describe the causes of reduced vision in this population. Methods: The visual acuity test was performed in 1688 school children of state public schools and in 611 school children of private schools. The children with visual acuity 0.7 or worse in one of the eyes were directed to a ophthalmic examination. Results: The reduced visual acuity prevalence in school children was $17.1 \%$ in state public schools, and $19.8 \%$ in private schools; this difference does not have statistical significance. The prevalence of children wearing eyeglasses was $2.4 \%$ in state public schools and $3.6 \%$ in private schools, 
without statistical significance between these results. The causes of reduced visual acuity were refractive errors, amblyopia, strabismus and other causes. Conclusion: The prevalence of reduced visual acuity in students of elementary state public and private schools was statistically the same, as well as the prevalence of children wearing eyeglasses. The causes of reduced visual acuity were refractive errors, amblyopia, strabismus, congenital cataract aphakia, zonular congenital cataract, persistent of the hyperplastic primary vitreous, optic nerve atrophy and macular disorder.

Keywords: Visual acuity; Cross-sectional studies; School health; Health education

\section{REFERÊNCIAS}

1. Temporini ER, Kara-José N, Taiar A. Validade da aferição da acuidade visua realizada pelo professor em escolares de $1^{\mathrm{a}}$ a $4^{\mathrm{a}}$ série de primeiro grau de uma escola pública do município de São Paulo, Brasil. Rev Saúde Pública 1977; 11:229-37.

2. Kara-José N, Temporini ER. Avaliação dos critérios de triagem visual de escolares de primeira série do primeiro grau. Rev Saúde Pública 1980;14:205-14.

3. Constanti FU, Costa MS, Salgado MB, Bastos CLFM, Benchimol E. Projeto de oftalmologia sanitária escolar. Rev Bras Oftalmol 1998;47:307-10.

4. Suzuki CK, Osawa A, Amino CJ, Yasmashita CH, Matuda E et al. Saúde ocular de alunos de primeira a oitava séries do primeiro grau de escolas estaduais de São Paulo, SP - 1992. Rev Bras Saúde Esc 1992;2:193-7.

5. Figueiredo RM, Santos EC, Almas de Jesus IA, Castilho RM, Santos EV. Proposição de procedimento de detecção sistemática de perturbações oftalmológicas em escolares. Rev Saúde Pública 1993;27:204-9.

6. Robinson B, Bobier WR, Martin E, Bryant L. Measurement of the validity of a preschool vision screening program. Am J Public Health 1999;89:193-8.

7. Conselho Brasileiro de Oftalmologia. Campanha nacional de reabilitação visual: manual de orientação. São Paulo: Imprensa Oficial do Estado; 1999.

8. Kara-José N, Holzchuh N, Temporini ER. Vícios de refração em escolares da cidade de São Paulo, Brasil. Bol Oficina Sanit Panam 1984;96:326-33.

9. Palazzo Neto G, Lauretti Filho A, Romão E. Estudo comparativo entre as medidas de acuidade visual feitas por um oftalmologista e uma atendente de enfermagem. Rev Bras Oftalmol 1992;51:45-9.

10. Sperandio AMG. Capacitação de professores e pajens para detecção precoce de problemas visuais; relato de uma experiência. Rev Bras Saúde Esc 1990;1:22-5.
11. Sloane AE, Rosenthal P. School vision testing. Arch Ophthalmol 1960; 64:763-70.

12. Gasperin Jr P, Bonilha MN, Castro \& Souza, G. Acuidade visual e visão cromática em escolares de uma escola de primeiro grau em Curitiba. Curitiba (PR): UFPR; 1985.

13. Temporini ER. Programas de prevenção da cegueira: participação da escola. Rev Bras Saúde Esc 1992;2:41-3.

14. Ottaiano JAA, Uras R, Melamed J. Midriáticos e cicloplégicos In: Lima ALH, Melamed J, Calixto N. Terapêutica clínica ocular. São Paulo: Roca;1995;19-27.

15. Negrel AD, Maul E, Pokharel GP. Refractive error study in children: sampling and measurement methods for a multi-country survey. Am J Ophthalmol 2000;129:421-2

16. Schimiti RB, Costa VP, Gregui MJF et al. Prevalence of refractive errors and ocular disorders in preschool and schoolchildren of Ibiporã - PR, Brazil (1989 to 1996). Arq Bras Oftalmol 2001;64:379-84.

17. Wright MC, Colville DJ, Oberklaid F. Is community screening for amblyopia possible, or appropriate? Arch Dis Child 1995;73:192-5.

18. Schellini SA, Shimoda GA, Hisano C. Estudo da prevalência das afecções oculares nos pré-escolares da cidade de Botucatu - São Paulo. Rev Bras Oftalmol 1987;46:21-6.

19. Degrazia JEC, Pellin JO, Silva, RA. Saúde escolar: levantamento das deficiências visuais mais freqüentes em população escolar de Porto Alegre. Rev Amrigs 1986;30:231-3.

20. Macchiaverni Filho, N. et al. Levantamento oftalmológico em escolares da primeira a quarta séries do primeiro grau na cidade de Paulina. Arq Bras Oftalmol 1979;42:289-94.

21. Guerrero VR, Martinez CCE, Wooley L. Defectos de refracción y rendimiento académico en la escuela primaria. Colombia Méd 1989;20:8-10.

22. Lauretti Filho, A.; Romão E. Estudo da acuidade visual e dos vícios de refração em crianças com baixo rendimento escolar. Rev Bras Oftalmol 1982;41:31-6.

23. Suzuki CK, Santos Neto, E. Estudo da acuidade visual de escolares da rede estadual e da rede particular de ensino do município de Barueri - SP, 1991. Rev Bras Saúde Esc 1994;3:244-51.

24. Maul E, Barroso S, Munoz SR. Refractive error study in children: results from la Florida, Chile. Am J Ophthalmol 2000;129:445-54.

25. Pastorino N, Penerini Y. Programa de detección de déficit de la agudeza visual en escolares sin patologia ocular aparente. Arch Argent Ped 1998;96:236-41.

26. Kara-José N, Carvalho KMM, Caldato R. Atendimento de amblíopes e prevalência na população pré-escolar, Campinas, São Paulo, Brasil. Bol Oficina Sanit Panam 1984;96:31-7.

27. Castro RS. Triagem visual e assistência oftalmológica em pré-escolares da cidade de Limeira (SP), 1995. Campinas (SP): UNICAMP 1997.

28. Adam Netto NA, Werner A, Rosa EL. Deficiência da acuidade visual em préescolares. ACM Arq Catarin Med 1999;22:193-6.

\section{An enviar un artigjo para publicação,

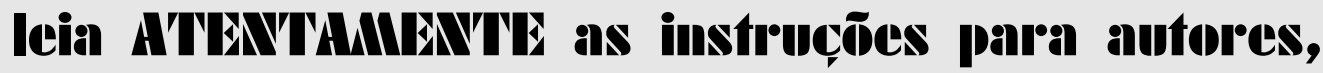 constante no final de cada fascículo.}

